\title{
Fortalezas y debilidades de un programa para el desarrollo de competencias genéricas
}

Sabino E. Rivero(1), Rodolfo F. Schmal(2) y Cristian L. Vidal-Silva(3)

(1) Escuela de Ingeniería Informática Empresarial, Facultad de Economía y Negocios, Universidad de Talca, Campus Lircay, Avenida Lircay S/N, Talca - Chile (e-mail: srivero@utalca.cl)

(2) Escuela de Ingeniería Civil Industrial, Facultad de Ingeniería, Universidad de Talca, Campus Curicó, Camino a Los Niches Km. 1, Curicó - Chile (e-mail: rschmal@utalca.cl)

(3) Departamento de Administración, Facultad de Economía y Administración, Universidad Católica del Norte, Antofagasta

- Chile. (e-mail: cristian.vidal@ucn.cl)

Recibido Jul. 5, 2019; Aceptado Ago. 28, 2019; Versión final Oct. 23, 2019, Publicado Ago. 2020

\begin{abstract}
Resumen
El objetivo de este trabajo es presentar los aspectos positivos y negativos, así como posibles mejoras, de un programa de formación en competencias genéricas en las carreras de pregrado de la Universidad de Talca (Chile). Se han desarrollado tres versiones del programa: PFF1 (programa de formación fundamental 1), PFF2 y PFF3. Esta es una investigación descriptiva con base en: una revisión de la documentación oficial de la Universidad de Talca, la aplicación de una encuesta y antecedentes recogidos por los autores. Los resultados muestran un proceso de mejoramiento continuo y de maduración expresado en una clarificación del concepto de competencia y una significativa reducción del tamaño del programa respecto del inicial. También muestran un intento por articular el programa con los componentes de formación básica, profesional o disciplinaria de cada carrera de pregrado. No obstante, se observa que aún existe espacio para significativas mejoras si se aspira a su consolidación.
\end{abstract}

Palabras clave: competencias; competencias transversales; competencias blandas; educación de pregrado; formación fundamental

\section{Strengths and weaknesses of a program for the development of generic competences}

\begin{abstract}
The objective of this paper is to present the strengths and weaknesses of a training program in generic competences in undergraduate degrees at the University of Talca (Chile). There are three versions of the program: fundamental formation program 1 (FFP1), FFP2, and PFF3. The present study is a descriptive research study that is based on a review of official documentation from the University of Talca, a survey, and background information collected by the authors. The results show a process of continuous improvement and maturation that is expressed in the clarification of the concept of competence. There is also a significant reduction in the size of the program when compared to that at the start. In addition, there is an attempt to articulating the program's components of the basic, professional, and disciplinary education of each undergraduate major examined. However, there is still room for significant improvements to consolidate the program.
\end{abstract}

Keywords: competences; transversal competences; soft competences; undergraduate education; fundamental training 


\section{INTRODUCCIÓN}

Durante las últimas décadas en la educación superior chilena se ha producido un sostenido incremento en la cobertura, desde menos del $20 \%$ a un poco más del $40 \%$ entre los jóvenes en edad de cursar estudios superiores. En paralelo, se ha estado en presencia de un progreso científico-tecnológico sin precedentes, a nivel mundial, que está incidiendo tanto en el ámbito productivo como en el de la interacción social. Así, se está transitando desde una sociedad que a sus profesionales les exigía conocimientos específicos perdurables en el tiempo hacia una sociedad que está planteando exigencias que se relacionan más con el comportamiento que con los conocimientos, en un mundo inestable y cambiante (Villarroel y Bruna, 2014).

Este trabajo se enmarca en la Universidad de Talca, donde el $90 \%$ de la matrícula de pregrado es de estudiantes de los tres primeros quintiles según la distribución de ingresos de sus familias y que en su mayoría no tienen una formación profesional. De allí que la universidad asumió en el año 2005, la responsabilidad de reemplazar el modelo educativo tradicional basado en dos componentes responsables de la formación básica y disciplinaria, a un modelo educativo para el desarrollo de competencias diseñado con 3 componentes esenciales de formación: un programa de formación fundamental (PFF), un programa de formación básica (PFB) y un programa de formación disciplinar (PFD). El primero de ellos, el PFF, destinado a desarrollar en los estudiantes las competencias genéricas o transversales, tanto instrumentales para el trabajo académico, como personales y ciudadanos de los estudiantes. Esta decisión involucró a la totalidad de los programas de docencia de pregrado (carreras) existentes (Moyano et al., 2012).

Tal como señalan Päivikki et al. (2018), las competencias genéricas en la educación superior proporcionan diferentes habilidades, capacidades 0 atributos transferibles, genéricos y claves en un contexto laboral actual. Por ejemplo, Gourlay y Stevenson (2017) contextualizan la relevancia actual de la educación superior para el desarrollo de competencias profesionales y genéricas. Una clasificación de los modelos curriculares basados en las competencias genéricas a desarrollar, contempla 3 categorías (Villarroel y Bruna, 2014; VillardónGallego, 2015): a) modelos formativos diferenciados, en los que cada carrera selecciona las competencias genéricas más pertinentes que deben tener sus egresados, y diseña un plan de formación propio para el desarrollo de tales competencias; b) modelos formativos integrados, donde las competencias genéricas se desarrollan en los módulos responsables de integrarlas con las competencias específicas que cada carrera exige a sus egresados; y c) modelos formativos paralelos, donde el desarrollo de las competencias genéricas tiene lugar en un mismo conjunto de módulos (asignaturas) con independencia de las competencias específicas. La Universidad de Talca optó por este último modelo para todas sus carreras en consideración a (Moyano et al., 2012): a) la relevancia que la sociedad y las empresas están asignando a las competencias genéricas a los profesionales universitarios; b) la necesidad de enfrentar el desafío de impartir educación a estudiantes que mayoritariamente provienen de ambientes socioeconómicamente vulnerables que requieren una formación en competencias genéricas de las que muchos de ellos carecen; y c) el interés de la Universidad de Talca porque la totalidad de sus egresados sean identificados por tener un sello común con independencia de la carrera en que se han matriculado.

El objeto de estudio considerado es el PFF orientado al desarrollo de competencias genéricas como parte de todos los planes de estudios de los programas de pregrado que se imparten en la Universidad de Talca. El principal objetivo de este trabajo es identificar las fortalezas y debilidades de las distintas versiones del PFF, tanto en su diseño como en su implementación. Así, por la naturaleza del objeto de indagación y el objetivo perseguido, se asume una perspectiva eminentemente cualitativa mediante la metodología de investigación descriptiva, tanto para a) recopilar y analizar antecedentes provenientes de documentos oficiales resoluciones universitarias (en adelante RU), acuerdos y actas de Consejos Académicos-; b) aplicar encuestas a los estudiantes que ya han aprobado la totalidad de los cursos incluidos en el PFF, y c) recoger la experiencia de los autores como docentes universitarios. Este trabajo, como una extensión de Schmal et al. (2020), presenta nuevas muestras asociadas a dos carreras y resultados con el propósito ya señalado. En consecuencia, este trabajo es una investigación de carácter descriptivo destinada a identificar los cambios experimentados, junto con sus fundamentos, por un programa de desarrollo de competencias genéricas desde su creación hasta la fecha. Se destaca que no se aborda ni la forma -metodologías- en que se habrán de desarrollar tales competencias, ni la evaluación de su logro.

\section{COMPETENCIAS GENÉRICAS}

Para enfrentar los desafíos presentes y futuros, la educación superior y la educación en general están experimentando fuertes cambios como consecuencia de los turbulentos tiempos que se viven en un mundo global y conectado (Cheng et al., 2018; Rowe y Zegwaard, 2017; Gul et al., 2017; Aldowah et al., 2017; Chan, 2016). De allí que sus características habituales se encuentran en revisión, y muy probablemente lo seguirán estando por mucho tiempo. Así, en el campo de la docencia surge el enfoque por competencias. Si bien el concepto de competencia tiene un origen utilitarista, de satisfacción de las demandas del mercado, del mundo 
empresarial, también tiene una concepción ciudadana, de equidad, de justicia, que busca abordar aquellas competencias genéricas, blandas o transversales vinculadas a las competencias, entre otras, para trabajar en equipo, bajo presión, orientado a resultados; para dialogar, expresarse y comunicarse por escrito, en forma gráfica y oral, para reflexionar (Päivikki et al., 2018).

Los autores de este trabajo se manifiestan de acuerdo con el modelo educativo orientado al desarrollo de competencias adoptado por la Universidad de Talca en la medida que el concepto de competencia no se limite a un enfoque orientado a la formación de profesionales, sino que vaya más allá del saber hacer y actuar en las organizaciones en que se desempeñen. Interesan ciudadanos con competencias no solo para disponer de una sociedad más productiva, sino que más democrática, más tolerante, más crítica y más reflexiva (Yanaze y de Deus, 2014; López, 2016). Según López et al. (2016), el enfoque basado en competencias surge en EEUU en el ámbito de la capacitación laboral y en las prácticas con miras a acercar a los estudiantes al mundo laboral real. Con los profundos cambios económicos y tecnológicos que se desatan desde los años 70, el concepto empieza a adquirir relevancia e ir más allá de la formación técnica-profesional. Según el International Board of Standard for Training, Perfomance and Instruction este modelo educativo se focaliza en el rendimiento de los resultados del aprendizaje (IBSTPI, 2019). En Europa, el concepto de competencia cobra fuerza al amparo del Proceso de Bolonia, concebido como un proceso destinado a establecer un espacio europeo de educación superior que en lo esencial facilite la movilidad de los trabajadores por la vía del reconocimiento de titulaciones, el establecimiento de equivalencias y sistemas de transferencias entre distintas universidades (Pereira y Costa, 2017).

Un concepto de competencia generalizado y aceptado es el de "saber hacer en un contexto", donde lejos de entenderse simplemente como "hacer", requiere de conocimientos teóricos, prácticos o teórico-prácticos, afectividad, compromiso, cooperación y cumplimiento, todo lo cual debe expresarse y comprobarse en el desempeño (Chan et al., 2017). Según López (2016), toda competencia está asociada a una actuación integral capaz de articular, activar, integrar, sintetizar, movilizar y combinar distintos saberes (conocer, hacer y ser) para ejercer una profesión, realizar una actividad o una tarea. Por tanto, el concepto de competencia integra conocimientos, potencialidades, habilidades, destrezas, prácticas y acciones de diversa índole (personales, colectivas, afectivas, sociales, culturales) en diferentes escenarios de aprendizaje y desempeño. Por su parte, Muñoz-Martinez (2015) y Zapata (2015), afirman que la competencia se asocia con la acción, con su puesta en práctica, con el saber actuar no solo en ámbitos laborales definidos dentro de las organizaciones, sino que va más allá de ellas, en la sociedad en que nos desenvolvemos.

En la Universidad de Talca, su modelo educativo apunta a formar un profesional capaz de "saber actuar en un contexto particular, poniendo en juego los recursos personales y contextuales (incluyendo redes) para la solución de un problema específico, con un proceso de reflexión sobre lo que se está haciendo" (UTALCA, 2019). Las competencias genéricas suelen definirse por contraste con las competencias específicas, y por tanto, como aquellas que rebasan los límites de una disciplina, necesarias para un desempeño eficaz y eficiente, más allá de una profesión en particular, y relevantes para desempeñarse en distintos contextos y para el aprendizaje a lo largo de la vida (Villardón-Gallego, 2015). Para los efectos de este trabajo las competencias genéricas son aquellas que permiten actuar exitosamente en contextos: a) con insuficiente información y/o donde no existen reglas claramente definidas; b) con dosis de incertidumbre en las consecuencias de las decisiones, susceptibles de generar estrés; y/o c) con horizontes de tiempos de actuación indefinidos (Savaneviciene et al., 2014).

\section{PROGRAMA DE FORMACIÓN FUNDAMENTAL}

Para el desarrollo de las competencias la Universidad de Talca optó por una estructura curricular: a) organizada en base a módulos valorados en créditos SCT (Sistema de Créditos Transferibles), equivalentes a los créditos ECTS (European Credit Transfer System), donde cada crédito representa 27 horas de trabajo académico de un estudiante; b) planificada en base a cursos o módulos a ser impartidos en un semestre académico (18 semanas) o en un año académico (36 semanas); c) conformada en base a un máximo de 30 SCT por semestre y 60 SCT por año académico; y d) estructurada en base a 3 componentes: de formación fundamental, de formación básica y de formación profesional, disciplinaria o específica, donde el primero está destinado a desarrollar las competencias genéricas, también llamadas transversales o sociales (Moyano et al., 2012).

La implementación del PFF, desde su inicio, quedó bajo la responsabilidad de la Vicerrectoría de Docencia de Pregrado, con el apoyo de dos unidades: el Centro de Innovación y Calidad de la Docencia (CICAD) y de la Dirección de Tecnologías del Aprendizaje (DTA) con el propósito de acompañar a los docentes en los procesos de enseñanza-aprendizaje y de incorporación de nuevas tecnologías de información y comunicación (TICs) asociados al cambio curricular (Moyano et al., 2012). 
A continuación, se describe en forma esquemática la evolución histórica del PFF responsable del desarrollo de las competencias genéricas en la Universidad de Talca y cuyo detalle se encuentra en Schmal (2015).

\section{PFF 1.0}

Desde el año 2005, en los primeros dos años de todas las carreras de la universidad, se comenzó a impartir un PFF con 11 módulos de 4 SCT cada uno, un conjunto de igual número de competencias generales, llamadas fundamentales, agrupadas en 3 líneas -instrumentales, interpersonales y ciudadanas (RU082, 2005). El diseño del PFF en su primera versión se estructuró como un componente paralelo a la formación básica y disciplinar, independiente de las especificidades de cada carrera, y de los perfiles de los estudiantes que ingresan a cada carrera. Esto último, en razón del interés de la universidad porque todos sus egresados estén dotados de un sello en la posesión de las competencias genéricas que compromete el PFF. Hoy PFF1.0.

Durante el año 2010, luego de cuatro años desde el inicio de la primera versión (PFF 1.0) se propusieron cambios tanto en su diseño como en su implementación. En el ámbito del diseño: a) reducir el tamaño del PFF 1.0 (44 SCT) de modo de dejar espacio para que las competencias se puedan desarrollar en el marco de las correspondientes profesiones; b) clarificar el concepto de competencia, en particular su nivel de complejidad, amplitud y de desarrollo esperado; c) contextualizar las competencias genéricas a los requerimientos de cada carrera, o a grupos de carreras asociadas a una misma disciplina; d) considerar el perfil de ingreso de los estudiantes que contemple el nivel de desarrollo de las competencias genéricas que poseen; y e) desconcentrar el PFF a lo largo de las carreras.

En el ámbito de la implementación de estas mejoras del PFF 1.0 se consideraron los siguientes hitos: a) socializar el PFF 1.0 entre académicos y estudiantes de manera que los comprometa en su éxito; b) implantar el PFF 1.0 gradualmente para los efectos de recoger las buenas prácticas y corregir errores; c) incorporar a la planta docente permanente de la universidad a los profesores responsables del desarrollo de las competencias implicadas; d) crear los mecanismos institucionales formales para operacionalizar, monitorear y evaluar la continuidad del desarrollo de las competencias implicadas en el PFF; e) relacionar la unidad institucional encargada del desarrollo del PFF con las escuelas responsables de las carreras; f) integrar los módulos y competencias objetivo del PFF en los módulos de formación básica y disciplinar; y g) disponer de un claro liderazgo a nivel institucional.

\section{PFF 2.0}

A contar del año 2011 se comienza a aplicar una actualización de la versión PFF 1.0 que llamaremos PFF 2.0. Esta nueva versión, contenida en RU180 (2011), implicó: a) reemplazar las 11 competencias fundamentales por 5 competencias fundamentales; b) reducir el PFF, de 44 SCT a 32 SCT; y c) redistribuir el total de los 32 SCT desde los 2 primeros años a los 4 primeros años en los planes de formación de todas las carreras que contemplaba el PFF 1.0. Estos cambios dan cuenta de una significativa disminución de la incidencia del PFF en los planes de formación. Por otra parte, como consecuencia de la dispersión de los módulos que conforman el PFF, desde los dos primeros años a los cuatro primeros años de cada carrera, el peso de los módulos del PFF en el primer año de cada carrera disminuye del 40 al 20\% dentro de la carga académica de los estudiantes. A ello se agrega una importante baja en el número de competencias comprometidas, disminución que refleja una clarificación de las competencias implicadas, en el sentido que toda competencia debe expresarse de forma tal que sea fácilmente comprensible. Además, esta nueva versión del PFF reveló la imposibilidad de desarrollar cabalmente las 11 competencias implicadas en el PFF 1.0 en tan solo dos años.

Desde un punto de vista cualitativo, el PFF 2.0 abre espacio para que el desarrollo de las competencias fundamentales no sea solo responsabilidad de los módulos del PFF, sino que sea complementada por los módulos de los otros programas que conforman el plan de formación (formación básica y formación disciplinaria); y para que las competencias fundamentales vayan siendo desarrolladas a lo largo de la carrera.

\section{PFF 3.0}

Para pasar a una fase de consolidación y de articulación del modelo de formación basada en competencias, en el año 2013, la Universidad de Talca analizó los perfiles profesionales demandados por los empleadores, expresados en términos de competencias, junto con un análisis de las conclusiones emanadas de un informe de evaluación del progreso en las competencias fundamentales (Canales, 2013). Este análisis permitió observar la necesidad de integrar las competencias fundamentales con las disciplinas asociadas a las distintas profesiones. Es así como en el año 2014 se reformula el PFF 2.0 para dar origen al PFF 3.0 (RU224, 2014) caracterizado por: a) centrarse en la formación de 3 competencias fundamentales: comunicación oral y escrita, 
integración a equipos de trabajo; y discernimiento en distintos ámbitos; b) estructurarse en 8 módulos con un total de 24 SCT; y c) asignarse 8 SCT a las escuelas responsables de las carreras que imparten con el propósito de desarrollar las competencias fundamentales en el contexto de las correspondientes profesiones.

Si bien las competencias genéricas que están demandando las empresas, y la sociedad en su conjunto, van más allá de estas 3 competencias, la Universidad decidió que el PFF se concentrase en ellas como expresión de su interés por que todos sus egresados se distingan por tener una sólida formación en tales competencias. Lo anterior no excluye la posibilidad de que cada carrera opte por complementarlas con otras competencias genéricas que consideren relevantes para el ejercicio profesional de sus egresados, cuya formación se integra ya sea en los componentes de formación básica o disciplinar.

EI PFF 3.0 da cuenta de que la formación de competencias fundamentales no estaba apropiadamente contextualizada, a pesar de que la reducción de 12 SCT del PFF 2.0 respecto del PFF 1.0 apuntaba en esa dirección. De allí que esta nueva versión, actualmente vigente dentro de los distintos planes de formación de los estudiantes en la Universidad de Talca, busca esencialmente articular la formación fundamental con las distintas disciplinas comprometidas en las carreras.

\section{RESULTADOS}

Comparando el PFF 3.0 respecto a sus versiones anteriores se observa que: a) se disminuye el total de créditos (SCT) primero de 44 a 32 SCT, y luego a 24 SCT, lo que implica una reducción casi en un 50\% respecto de la primera versión del PFF; b) se desconcentra el peso de los módulos del PFF 1.0 desde los dos primeros años de cada carrera a los cuatro primeros años de todas las carreras; c) disminuye significativamente el número de competencias cuyo desarrollo comprometía el PFF 1.0 desde 11 competencias en su primera versión, a 5 competencias en el PFF 2.0, y luego a 3 competencias en la versión actual el PFF 3.0; y d) se traspasan 8 SCT del PFF 2.0 a las Escuelas para la articulación de las competencias comprometidas en el PFF en el marco de cada carrera.

Para recoger la opinión de los estudiantes, a fines del mes de abril del año 2019 se aplicó una encuesta a 69 estudiantes matriculados en las carreras de Ingeniería Civil Industrial e Ingeniería en Informática Empresarial, y que a la fecha han aprobado la totalidad de los módulos comprometidos en el PFF 3.0, esto es, que han completado el PFF en su versión actual. Esta encuesta tuvo como objetivo registrar los aspectos positivos y negativos que consideran posee el PFF 3.0 junto con las mejoras sugeridas a la luz de la experiencia que han tenido.

Transcurridos cinco años desde la implementación de la versión PFF 3.0, se aplicó un cuestionario elaborado bajo un criterio cualitativo con preguntas relacionadas con los aspectos positivos, negativos y a mejorar en el PFF. Las dos primeras preguntas cerradas, a partir de respuestas obtenidas en idéntico cuestionario aplicado en años anteriores, con opción de incorporar opciones adicionales, marcándose si/no para cada alternativa. Las respuestas a la tercera pregunta asociada a los aspectos a mejorar se dejaron abiertas. Los resultados se condensan en las tablas 1,2 y 3.

Las dos primeras incluyen los aspectos positivos y negativos que los estudiantes resaltan del PFF en su versión actual, y la tercera, las mejoras sugeridas.

Tabla 1: Aspectos positivos destacados por los estudiantes del pff 3.0

\begin{tabular}{|l|c|c|}
\hline \multicolumn{1}{|c|}{ Aspectos positivos } & $\begin{array}{c}N^{\circ} \text { de } \\
\text { respuestas }\end{array}$ & $\begin{array}{c}\% \text { del total de } \\
\text { estudiantes }\end{array}$ \\
\hline Complementa la formación profesional facilitando el desarrollo de habilidades blandas & 54 & $78 \%$ \\
\hline Estimula el trabajo en equipo y contribuye a la interacción entre equipos de trabajo & 50 & $72 \%$ \\
\hline Refuerza la necesidad de un comportamiento ético y moral & 44 & $64 \%$ \\
\hline Existencia de módulos con alumnos de diferentes escuelas & 39 & $57 \%$ \\
\hline
\end{tabular}

Respecto de los elementos positivos, en la tabla 1 se destaca que el $78 \%$ de los estudiantes considera que el PFF contribuye a su formación profesional haciéndose cargo del desarrollo de las competencias blandas que compromete. En un segundo lugar, el $72 \%$ de los estudiantes valora que el PFF estimule el trabajo en equipo y contribuya a la interacción entre distintos equipos de trabajo, anticipando de este modo una realidad con la que se enfrentarán en su futuro laboral. También se destaca que el $64 \%$ de los estudiantes estiman 
que el PFF refuerce el comportamiento ético-moral en el ámbito de las decisiones y las actuaciones. Por último, el $57 \%$ de los estudiantes estima positivamente que los cursos del PFF estén conformados por estudiantes de diferentes carreras, lo que les permite relacionarse con estudiantes que poseen otras maneras de observar y abordar un mismo problema.

En cuanto a los aspectos negativos del PFF 3.0, en la tabla 2 se observa que el $72 \%$ de los estudiantes considera que las clases son poco dinámicas, careciendo de atractivo por ser eminentemente teóricas. Por otra parte, el $49 \%$ de los estudiantes estima que el PFF incluye módulos y/o materias innecesarias -sin especificar cuáles-. Con porcentajes que varían entre el $40 \%$ y $45 \%$, a) los estudiantes encuestados objetan los horarios que tienen los módulos del PFF, tanto por su falta de flexibilidad como por su falta de coherencia con los horarios de cada carrera y/o alumno; b) que los módulos exigen mucho tiempo de trabajo autónomo, esto es, de trabajo fuera de aula; y c) la obligatoriedad de cursar todos los módulos que conforman el PFF.

Tabla 2: Aspectos negativos destacados por los estudiantes del pff 3.0

\begin{tabular}{|l|c|c|}
\hline \multicolumn{1}{|c|}{ Aspectos negativos } & $\begin{array}{c}\mathrm{n}^{\circ} \text { de } \\
\text { respuestas }\end{array}$ & $\begin{array}{c}\% \text { del total } \\
\text { de estudiantes }\end{array}$ \\
\hline Contiene clases teóricas que hacen a las clases poco dinámicas/atractivas & 50 & $72 \%$ \\
\hline Incluye módulos/materias innecesarias & 34 & $49 \%$ \\
\hline Tiene malos horarios & 31 & $45 \%$ \\
\hline Demanda mucho tiempo de trabajo autónomo & 30 & $43 \%$ \\
\hline Posee un carácter obligatorio cuando no necesariamente todos lo requieren & 28 & $41 \%$ \\
\hline
\end{tabular}

En la tabla 3 se observan las principales acciones que los estudiantes consideran que se deben realizar para mejorar el PFF. Al respecto proponen desarrollar actividades más prácticas que teóricas, y que las clases tengan un sentido más lúdico. También invitan a integrar módulos, revisar sus contenidos, o asignar un $n^{\circ}$ de créditos más ajustado a la realidad, revisando los contenidos de los módulos, o mejorando la asignación de empresas donde los estudiantes han de desarrollar los proyectos. Todo ello como una manera de superar la excesiva carga de trabajo autónomo, expresada como la demanda de tiempo por parte de los módulos del PFF. Por otra parte los estudiantes proponen flexibilizar horarios para compatibilizarlos con los que poseen en sus respectivas carreras, así como contextualizar los contenidos del PFF al ámbito profesional de las carreras. También recomiendan efectuar un diagnóstico inicial respecto de las competencias que traen los estudiantes con miras a levantar la exigencia de cursar todos los módulos contenidos en el PFF. Junto con ello se sugiere levantar la obligatoriedad de la asistencia que se exige actualmente. Los aspectos a mejorar mencionados por los estudiantes que se incluyen en la tabla 3, en su mayoría tienden a reforzar los aspectos positivos del PFF señalados en la tabla 1, o bien, a reducir, acotar, o eliminar los aspectos negativos incluidos en la tabla 2.

Tabla 3: Aspectos a mejorar destacados por los estudiantes del pff 3.0

Desarrollar más trabajos prácticos en clases, que las hagan menos teóricas y más lúdicas

Integrar módulos y/o disminuir tiempos de trabajo autónomo y/o mejorar la asignación de créditos

Tener horarios más apropiados y/o flexibilizar horarios

Contextualizarlos al ámbito profesional de las carreras

Efectuar un diagnóstico inicial que permita a los alumnos eximirse en algunos módulos del PFF

Levantar la obligatoriedad de asistencia

\section{DISCUSIÓN}

Una revisión de la documentación de las organizaciones colegiadas internas de la universidad -consejos de docencia, de escuelas, de facultades y académico- desde la implementación del PFF permite verificar la presencia de un proceso de mejora continua caracterizado por 1). Una reducción del tamaño del PFF (44 SCT) para abrir espacio a la contextualización de las competencias asociadas a la formación básica y/o la formación disciplinar; 2). Una articulación de las competencias genéricas a los requerimientos de cada carrera, o a grupos de carreras de una misma disciplina; 3). Una distribución del PFF durante la formación de las carreras en base el nivel de madurez y requerimientos prácticos de las competencias genéricas implicadas; y 4). Una clarificación del concepto de competencia, en particular su nivel de complejidad, amplitud y de desarrollo esperado. Así, si bien, actualmente existe un mayor conocimiento del concepto de competencia 
que al inicio, aún faltaría especificar el nivel de complejidad y amplitud esperado para cada una de las competencias genéricas que el PFF 3.0 aspira desarrollar. Cabe señalar que, a la fecha, los perfiles de egreso de las distintas carreras no especifican el nivel de desarrollo de las competencias genéricas a alcanzar. De esta forma, implícitamente la universidad deja en libertad de acción a cada carrera o escuela la definición del nivel de desarrollo que aspira que posean sus egresados en las competencias genéricas de las que el PFF 3.0 es responsable. Ello significaría que el grado de desarrollo de las competencias genéricas comprometidas sería a un nivel básico que se requeriría definir con precisión.

La casi totalidad de los aspectos negativos reportados, en los que destacan la exigencia de un excesivo trabajo autónomo y la inclusión de módulos/materias poco necesarias, da cuenta de estudiantes que valoran más las competencias de la formación básica y disciplinar, que aquellas incluidas en el PFF 3.0. Entonces, existe un desafío no menor por lograr que los estudiantes perciban con mayor claridad que su futuro, en un contexto de desarrollo científico-tecnológico que tiende a la automatización/robotización, no depende tan solo de su formación básica y disciplinar, sino que de poseer las competencias genéricas que diferencian a las personas. En general, si bien se observan avances a más de una década del inicio del PFF, a la fecha aún no se encuentra sólidamente socializado entre estudiantes y docentes. De hecho, el PFF surgió al comienzo del presente siglo a raíz de una preocupación de las autoridades superiores de la universidad por factores tanto internos (masificación de la educación superior) como externos (globalización, nuevas tecnologías de información y comunicación) sin participación estudiantil ni de los docentes.

Por otra parte, si bien existe una institucionalidad de apoyo a la implementación del PFF, ésta ha sufrido múltiples avatares expresados en cambios de dependencia, de autoridades y profesores del programa. En efecto, a lo largo del tiempo el PFF ha estado bajo distintas dependencias, con una alta rotación en su dirección, al igual que en las unidades de apoyo (CICAD y DTA) al igual que en sus docentes, la mayoría contratados a tiempo parcial por horas. Además, aun cuando actualmente se incluyen 8 SCT del PFF dentro de los módulos de formación básica y disciplinaria, esta integración o articulación no ha estado exenta de dificultades con disímiles resultados en las distintas carreras. A lo expuesto, se agrega la ausencia de un claro liderazgo institucional de apoyo a la implementación del PFF que sea visible tanto por los profesores y estudiantes, apoyo que se exprese no solo a nivel discursivo, sino que de forma concreta en provisión de los recursos, particularmente del tiempo docente que demanda un modelo educativo de esta naturaleza.

A la luz de la evolución del PFF, que demuestra la complejidad de incorporar competencias genéricas en los programas de formación universitaria, los autores proponen aplicar una reingeniería con la participación de los actores directamente involucrados (estudiantes, profesores y egresados), para plantear que el desarrollo de las competencias genéricas sea parte de los módulos de formación básica y disciplinaria. La actual demanda de la sociedad por dichas competencias invita a revisar planes de formación en las casas de estudios superiores que resuelvan optar por este enfoque. En tal sentido, este trabajo presenta la versión actual del PFF en la Universidad de Talca, y la trayectoria seguida desde su origen, como una base susceptible de mejoras, para instituciones de educación y sus respectivas carreras que ya están incursionando, o están por incursionar, en modelos curriculares orientados al desarrollo de competencias.

\section{CONCLUSIONES}

Las vicisitudes experimentadas por el PFF en sus distintas versiones (2005-2013) en la Universidad de Talca parecen explicarse esencialmente por su carácter pionero en Chile, como porque en su momento no se tenía la capacidad y experiencia que sí existe en la actualidad. Lo más relevante del actual PFF 3.0 es que reduce y clarifica significativamente las competencias que compromete, redistribuye sus módulos dentro del plan de estudios, abriendo espacio a su articulación y contextualización por parte de los académicos de cada carrera y mantener la identidad común que se aspira tengan los estudiantes al momento de egresar. Sin embargo, habiendo transcurrido ya más de una década desde el inicio de la implementación del PFF 1.0, los estudiantes continúan sin visualizar la importancia y utilidad de los módulos que conforman el PFF y que están destinados a proveer las competencias genéricas que la sociedad moderna demanda a sus profesionales.

\section{REFERENCIAS}

Aldowah, H., Rehman, S., Ghazal, S. y Umar, I., Internet of Things in Higher Education: A Study on Future Learning, Journal of Physics Conference Series, vol. 892, pp 012 - 017 (2017)

Canales, T., Informe de Evaluación del Progreso de la Cohorte 2010, Centro de Psicología Aplicada, Facultad de PsicologíaUniversidad de Talca, Talca - Chile (2013)

Chan, R., Understanding the Purpose of Higher Education: An Analysis of the Economic and Social Benefits for Completing a College Degree, Journal of Education Policy, Planning and Administration, Vol. 6(5), 1-40 (2016) 
Chan, C., Fong, E., Luk, L. y Ho, R., A Review of Literature on Challenges in the Development and Implementation of Generic Competencies in Higher Education Curriculum, International Journal of Educational Development, Vol 57, 110 (2017)

Cheng, M, Lee, K. y Chan, C., Generic Skills Development in Discipline-Specific Courses in Higher Education: A Systematic Literature Review, Curriculum and teaching, Vol 33 (2), 47 -65 (2018)

Gourlay, L. y Stevenson, J., Teaching Excellence in Higher Education: Critical Perspectives, Teaching in Higher Education, Taylor \& Francis Group, 22(4), 391 - 395 (2017)

Gul, S., Asif, M. y otros 5 autores, A Survey on Role of Internet of Things in Education, IJCSNS International Journal of Computer Science and Network Security, Vol.17 No.5, pp. 159-165 (2017)

IBSTPI, International Board of Standards for Training, Performance and Instruction, (en línea), https://www.ibstpi.org/. Acceso: 09 de Mayo (2019)

López, C., Benedito, V. y León, M.J., El Enfoque de Competencias en la Formación Universitaria y su Impacto en la Evaluación. La Perspectiva de un Grupo de Profesionales Expertos en Pedagogía, http://dx.doi.org/10.4067/S071850062016000400003, Formación Universitaria, Vol. 9(4), 11-22, La Serena, Chile (2016)

López, E., En Torno al Concepto de Competencia: un Análisis de Fuentes, Revista de Currículum y Formación del Profesorado, Vol. 20 (1), 311 - 322, Enero - Abril (2016)

Moyano, E., Vásquez, M. y Faúndez, F., El Modelo Educativo de la Universidad de Talca, Vicerrectoría de Docencia de Pregrado, Universidad de Talca, Chile (2012)

Muñoz-Martínez, M. y Garay-Garay, F., La investigación como forma de desarrollo profesional docente: Retos y perspectivas, , Estudios Pedagógicos, Vol. 41(2), 389 - 399, Valdivia, Chile (2015)

Päivikki, J., Seija, N. y Päivi, T., Models for the Development of Generic Skills in Finnish Higher Education, https://doi.org/10.1080/0309877X.2016.1206858, Journal of Further and Higher Education, Vol. 42 (1), 130-142 (2018)

Pereira, O. y Costa, C.A., The Importance of Soft Skills in the University Academic Curriculum: The Perceptions of the Students in the New Society of Knowledge, http://dx.doi.org/10.18533/ijbsr.v7i6.1052, International Journal of Business and Social Research, Vol 7 (3), 25 - 34 (2017)

Rowe, A.D. y Zegwaard, K.E., Developing Graduate Employability Skills and Attributes: Curriculum Enhancement Through Work-Integrated Learning, Asia-Pacific Journal of Cooperative Education, 18(2), 87-99 (2017)

RU082, Promulga Acuerdo $n^{\circ} 734$ de la Junta Directiva que Aprueba la Propuesta del Perfil Genérico y la Estructura Curricular del Grado de Licenciado y del Título Profesional contenido en la Resolución Universidad de Talca № 082 del 18 de enero (2005)

RU180, Promulga Acuerdo n 1151 de la Junta Directiva que Aprueba la Propuesta de Cambios al Programa de Formación Fundamental contenido en la Resolución Universidad de Talca Nº 180 del 14 de marzo (2011)

RU224, Promulga acuerdo $n^{\circ} 1384$ de la Junta Directiva que Aprueba la Propuesta de Modificación del Programa de Formación Fundamental contenido en la Resolución Universidad de Talca № 180 del 28 de febrero (2014)

Savaneviciene, A., Rutelione, A. y Ciutiene, R., Crucial Transversal Competences in the Changing Environment: Case of the European SMES Managers, https://doi.org/10.5755/j01.em.19.1.5485, Economics and Management, 19(1), 100-108 (2014)

Schmal, R. Evolución de un Programa de Formación en Competencias Genéricas, http://dx.doi.org/10.4067/S071850062015000600012 , Formación Universitaria, Vol. 8 (6), 95 - 106 (2015)

Schmal, R., Rivero, S. y Vidal-Silva, C., "El desafío de construir un programa para el desarrollo de competencias genéricas: un estudio de caso ", Educação e Pesquisa, Brasil, (por aparecer), vol. 46 (2020)

UTALCA, Universidad de Talca, Evaluación del Programa de Formación Fundamental - Acuerdos № 1206,1208 y 1614 del Consejo Académico Extraordinario 2010 y 2014, (en línea), https://www.utalca.cl. Acceso: 09 de Mayo (2019)

Villardón-Gallego, L., Competencias Genéricas en Educación Superior, Narcea Ediciones, España, 192 (2015)

Villarroel, V. y Bruna, D., Reflexiones en Torno a las Competencias Genéricas en Educación Superior: Un Desafío Pendiente, http://dx.doi.org/10.5027/psicoperspectivas-Vol13-Issue1-fulltext-335, Psicoperspectivas, Vol. 13 (1), 23 - 34 (2014)

Yanaze, L. y de Deus, R., Transversal Competencies of Electrical and Computing Engineers Considering Market Demand, Frontiers in Education Conference (FIE), IEEE, Madrid - España, 1 - 4 (2014)

Zapata, J., El Modelo y Enfoque de Formación por Competencias en la Educación Superior: Apuntes sobre sus Fortalezas y Debilidades, https://doi.org/10.18359/ravi.1420, Revista Academia y Virtualidad, Vol. 8 (2), 24 - 33 (2015). 